\title{
Acute fatty liver of pregnancy: A case report of successfully managed an uncommon catastrophic disease.
}

\author{
Varuni $K^{1}$, Sivansuthan $S^{1,}$ Gajanthan $R^{1}$ \\ ${ }^{1}$ Teaching hospital Jaffna.
}

\section{Background}

Acute fatty liver disease of pregnancy has universally high fatality rate (1). But with modern treatment and early anticipation of diagnosis and complication, and with appropriate supportive management it has improved prognosis (2). AFLP also known as acute hepatosis of pregnancy/ acute yellow atrophy of the liver (3) It is usually a diagnosis of exclusion and a strong index of suspicion can lead to timely diagnosis. Delay in diagnosis is associated with morbid complications with high mortality. Liver biopsy is the gold standard for diagnosing AFLP, but due to the presence of coagulation abnormalities diagnosis is usually made by clinical and laboratory findings (4). Even though pathophysiology is unknown it is believed to be caused by microvascular stetosis of liver cells due to impaired long chain fatty acids metabolism in mitochondria of the fetus $(2,5)$. It affects mainly first pregnancy, thin patients and multiple pregnancies and male fetus $(4,5)$. It can recur in subsequent pregnancies (5).

\section{Case report}

A 30-year-old $\mathrm{P}_{2} \mathrm{C}_{0}$ mother with twin pregnancy presented at her POA $36+6$ weeks. Her first pregnancy was a miscarriage at 12 weeks of POA. Her antenatal history was unremarkable. She was admitted to a local Hospital with 3 days fever and cough and treated as chest infection. She developed nausea, vomiting and giddiness. Urgent USS scan showed one twin had died. She was urgently transferred to Teaching hospital, Jaffna.

On admission her BP was 140/90mmhg and pulse rate $125 \mathrm{bpm}$. She was icteric and had bilateral mild ankle edema and tender right hypochondrium. Lungs were clear. There was no evidence of rupture of membrane/ or PV bleeding. Fetal heart rate was monitored showed fetal distress. Her investigations revealed, WBC 16000, platelet 125,000, Hb $11 \mathrm{~g} /$ dl, INR 1.6, APTT 85.6, AST 290 U/L, ALT 310 $\mathrm{U} / \mathrm{L}$, albumin $22 \mathrm{~g} / \mathrm{dl}$, total bilirubin $143 \mathrm{umol} / 1$ with direct component 94umol/1, creatinine $282 \mathrm{mg} / \mathrm{dl}$ and normal UFR. Repeat USS also confirmed that only single viable fetus with signs of fetal distress. Our main differential diagnosis was acute fatty liver of pregnancy/ sepsis and DIC secondary to IUD.

She was transferred to ICU next day. Broad spectrum antibiotics were started. Blood picture showed early DIC. She underwent LSCS under spinal anesthesia with intraoperative FFP and cryoprecipitate. Both fetuses were stillborn without maceration. In the immediate postpartum she developed restlessness, confusion and she deteriorated. There was ongoing mild PV bleeding. She was intubated and vertilated. Liver failure and anti-coma regime was started.

USS abdomen showed severe fatty liver changes. Liver enzymes rose up to $400 \mathrm{U} / \mathrm{L}$ and AST was more than ALT. INR was persistently high with maximum of 3.3 and bilirubin rose to $365 \mathrm{umol} / \mathrm{l}$. Platelet dropped to 10,000 and $\mathrm{Hb}$ to $5.3 \mathrm{~g} / \mathrm{dl}$. Creatinine reached to $450 \mathrm{umol} / \mathrm{l}$. ABG showed metabolic acidosis. TSH, ANA and Hepatitis screening were normal. Cultures, DAT, ECHO were normal. Repeat blood picture showed MAHA. CT brain scan thrice was normal. CXR showed ARDS. Her $\mathrm{O}_{2}$ requirement was $90 \% \mathrm{O}_{2}$ with $\mathrm{SPO}_{2} 68 \%$ indicating severe ARDS.

She was given factor VII, FFP, Cryo, platelets and blood with the advice of hematologist. She was treated with Mannitol, Human albumin and NAC infusion as suggested by gastroenterologist. Hypoglycemia was corrected with $10 \%$ and $25 \%$ dextrose infusion. Fluid status was optimized with CVP guidance. Blood pressure was supported by 4 inotropes, first Noradrenalin and then Dopamine, Dobutamine and Vasopressine. Her prolonged ventilation was supported with tracheostomy. She was initially offered peritoneal dialysis and later 5 cycle of haemodialysis for anuric renal failure after discussing with Nephrologist. As there was no improvement she underwent total plasma exchange 5 cycles every other day. Finally, her GCS 
and biochemical parameters become normalized. She was weaned off from ventilator after $3 / 52$. Her ICU stay was complicated with hospital acquired pneumonia, catheter sepsis, critical illness myopathy, bed sores and depression. After rehabilitation for 2 more weeks she was discharged home.

\section{Discussion}

Acute fatty liver of pregnancy (AFLP) affects 1 in 7000 to 1 in 16000 deliveries $(1,5)$. Due to the rapid diagnosis tools and speedy delivery the maternal mortality rate has decreased from $80-85 \%$ to $7-18 \%$ and the fetal mortality rate from $50 \%$ to $9-23 \%$ (4). Early detection and anticipation of complication are the most important management step in successful outcome in AFLP even though patient developed life-threatening complications. But in this patient, there is some delay in transfer and getting emergency delivery due to ICU availability and coagulation derangement in this patient.

In this case our Differential diagnosis was AFLP, HELLP and IUD with DIC. Severe coagulopathy, jaundice, hepatic encephalopathy, ascites, hypoglycemia, and a mild to moderate elevation of transaminase levels are the key features ofAFLP rather than HELLP (4). Also developing ARDS, DIC, HRS and encephalopathy strengthen the diagnosis of AFLP. As the first twin died in utero within 1 day it is also unlikely to cause DIC. To say HELLP there was no High BP or proteinuria.

Swansea criteria for diagnosing AFLP have been used if Six or more of the following features : vomiting; abdominal pain; polydipsia/polyuria; encephalopathy; elevated bilirubin $>14 \mu \mathrm{mol} / \mathrm{L}$; hypoglycemia $<4 \mathrm{mmol} / \mathrm{L}$; elevated urate $>340$ $\mu \mathrm{mol} / \mathrm{L}$; leukocytosis $>11 \times 10^{9} / \mathrm{L}$; ascites or bright liver on ultrasound; elevated transaminases; elevated ammonia $>47 \mu \mathrm{mol} / \mathrm{L}$; renal impairment creatinine $>150 \mu \mathrm{mol} / \mathrm{L}$; coagulopathy $(\mathrm{PT}>14 \mathrm{sec}$ or APTT $>34 \mathrm{sec}$ ), or micro vesicular steatosis on liver biopsy(4)(5). This patient easily completed this criterion.

Liver biopsy shows characteristic pictures of AFLP, micro vesicular fatty infiltration of the hepatocytes. The cytoplasm shows foamy appearance due to fat droplets around centrally located nuclei. The fatty infiltration spares zoon around portal tracts and involve only central and mid zones of the lobule (5). As it is invasive method it is rarely performed during pregnancy due to coagulopathy and hemodynamic instability.

Women who develop AFLP are more likely to have a heterozygous long-chain 3-hydroxyacylcoenzyme A dehydrogenase (LCHAD) deficiency due to inherited defects in mitochondrial beta oxidation of fatty acids. It can be present in either of fetus or mother $(5,6)$. LCHAD is found on the mitochondrial membrane and is involved in the formation of 3- ketoacyl-coA from 3 hydroxyacyl$\mathrm{CoA}$. This gene mutation is recessive; therefore, under normal conditions, women have normal fatty acid oxidation. The commonest mutation is G1528C (5). However, if the fetus is homozygous for this mutation, it will be unable to oxidize fatty acids. These metabolites products produced by the fetus is accumulated and passed to the mother, who, because of diminished enzyme function, cannot metabolize the additional fatty acids by maternal liver. This results in hepatic strain leading to the development of AFLP, which can be relieved by delivery of the infant $(5,6)$.

Total plasma exchange become key role in AFLP. Recent literatures reviews confirm that all patients showed remarkable improvement. If it is combined with CRRT it will give added success (6,7). It acts by removing pathologic substances and reducing the further damage. It can reverse the pathologic process. Factor VII also has been successfully used off label for control bleeding in DIC (5).

\section{Conclusion}

- AFLP should be a differential diagnosis in a pregnant patient present with icterus with vomiting and abdominal pain.

- Early termination of pregnancy would be the mainstay of treatment.

- Even though fetus delivered, patient can deteriorate further, and we should anticipate hepatic encephalopathy, hepatorenal shutdown, ARDS and DIC.

- Multiple specialty team approach and ICU care will influence the good outcome. 
- TPE has significant role in case of AFLP complicated with HRS and encephalopathy.

- Factor VII has significant role in AFLP with DIC.
Abbreviations
$\mathrm{DIC}=$ disseminated intravascular coagulation, $\mathrm{MAHA}=$ microangiopathy hemolytic anemia, HELLP $=$ Hemolysis, Elevated Liver enzymes, Low Platelet, IUD = Intra Uterine Death, HRS $=$ Hepato Renal Syndrome, ARDS= Acute Respiratory Distress Syndrome. DAT= Direct Agglutination Test.

\section{References}

1. Singh N, Meena P. Acute Fatty Liver of Pregnancy: ACase Report and Literature Review. J Preg Child Health 2015 jun; 2:e118
2. Camero DC. Acute fatty liver of pregnancy. Wikipedia. 2018.

3. Kalpana SV, Veena RS, Geeta PP. Acute fatty liver of pregnancy: A case report of an uncommon disease. Indian J Crit Care Med 2009 Jan-Mar;13(1):34-6.

4. Nihal AR, Abdullah AH, Fehmida Z. Atypical Case of Acute Fatty Liver of Pregnancy. Sultan Qaboos Univ Med J 2011 Nov;11(4):507-510.

5. Richard HL, Tram TT. Acute fatty liver of pregnancy. UpToDate; 2018 jul 2.

6. Michael JB, Ronald MR. Acute Fatty Liver of Pregnancy. Medscape; 2018 feb 14.

7. Chu YF, Meng M, Zeng J, Zhou HY, Jiang JJ, Ren $\mathrm{HS}$, et al. Effectiveness of combining plasma exchange with continuous Hemodiafiltration on Acute fatty liver of pregnancy complicated with multiorgan dysfunction. Artif organs.2012 jun;36(6):530-4. 